\title{
Cytological Diagnosis of Leiomyogenic Tumors of the Stomach
}

\author{
Akira Sato, Kuniharu Ishioka, Mitsuo Kobiyama, \\ Masashi Goto, Shigeru Asaki, Yoshihide Umetsu, Tokiaki \\ Toyohara, Michinori Sato, Kiyoaki Hanzawa, Kimko \\ Ogashiwa, Yoshio Goto, Haruki Wakasa,* Tetsutaro \\ Takeda, $\dagger$ Hisae Irumagawa, + Yukiko Tsuda $\ddagger$ and Atsuko \\ AIzAWA +
}

The Third Department of Internal Medicine, Tohoku University School of Medicine, Sendai 980, *the Second Department of Pathology, Tohoku University School of Medicine, Sendai 980, †Miyagiken Seijinbyo Center, Natori 981-12, and ${ }_{+}^{+}$the Cancer Detection Center of Miyagi Cancer Society, Sendai 980

Sato, A., Ishioka, K., Kobiyama, M., Goto, M., Asaki, S., Umetsu, Y., Toyohara, T., Sato, M., Hanzawa, K., Ogashiwa, K., Goto, Y., Wakasa, H., Takeda, T., Irumagawa, H., Tsuda, Y. and Aizawa, A. Cytological Diagnosis of Leiomyogenic Tumors of the Stomach. Tohoku J. exp. Med., 1980, 132 (2), 213-223 — The cytological findings in 7 cases of leiomyoma and 4 cases of leiomyosarcoma can be summarized as follows: For differential diagnosis of these lesions, in comparison with leiomyoma cells, leiomyosarcoma cells were found to have 1) increased minor axis diameters of the nuclei and nuclear anisokaryosis, 2) dark nuclear staining, 3) enlarged and darkly stained chromocenters, 4) dark staining and thickening of the nuclear rim, 5) enlargement of nuclear clear areas, 6) increased numbers of oval nuclei and greater pleomorphism, 7) an increase in size and number of the nucleoli, 8) a strong tendency for cell atypism, such as anisocytosis and pleomorphism. In the light of these findings, it is believed that differential diagnosis is indeed possible.—— cytology; gastric leiomyoma; gastric leiomyosarcoma

Despite remarkable advances in the diagnosis of stomach diseases, there are still major difficulties involved in the preoperative histological diagnosis of submucosal tumors of the stomach. This has been due to the fact that still no method for sampling submucosal tumors has been established and the fact that the incidence of such tumors is low, thereby preventing a statistical analysis of diagnostic and operative results. Nonetheless, in recent years due to the development of new biopsy methods, including thorny needle biopsy (Ishioka et al. 1979) and needle aspiration cytology (Soeda et al. 1976) using a fibergastroscope, it is thought that the possibilities for cytological diagnosis of submucosal gastric tumors have greatly improved.

We have made a comparative investigation of the cytological findings of the 
leiomyogenic tumors, the leiomyomas and the leiomyosarcomas, which are numerous among the submucosal tumors and whose malignancy determination is important. Discussion is also made concerning the possibility of differential diagnosis based upon cytological findings.

\section{Materiats AND Methods}

Materials included 11 cases of leiomyogenic tumor, 7 leiomyomas and 4 leiomyosarcomas, which were treated at the Tohoku University Hosptial and its afiliated institutions. Definitive histopathological diagnosis was made postoperatively in all cases. Using the cytological materials obtained by the direct smear method from the surgical specimens and at $\times 1000$ magnification with Papanicolaou staining, the cytological findings of 100 tumor cells from each (a total of 1100 cells) were examined.

\section{RESULTS}

The cytological findings of the leiomyomas and leiomyosarcomas are summarized in Table 1.

\section{Nuclear size}

The major axis diameter of the nuclei of the leiomyoma cells (hereafter LMC) measured $18.0 \pm 5.6 \mu \mathrm{m}$, whereas that of the leiomyosarcoma cells (hereafter LMSC) was $15.3 \pm 7.4 \mu \mathrm{m}$, or roughly by $3 \mu \mathrm{m}$ smaller than the LMC. There was less anisokaryosis in the LMC than the LMSC.

As shown in Fig. 1, the minor axis diameters of the nuclei of these cells were markedly different. That is, the average was $4.6 \pm 1.0 \mu \mathrm{m}$ for LMC, the great majority being between 3 and $8 \mu \mathrm{m}$. In contrast, the minor axis diameter of the LMSC was $7.5 \pm 2.7 \mu \mathrm{m}$ with a range between 3 and $18 \mu \mathrm{m}$. With regard to the correlation between the type of lesion and nuclear size, it was found that the incidence of cells with minor axis greater than $5 \mu \mathrm{m}$ was less than $40 \%$ in all of the LMC, averaging only $25.7 \%$, whereas it was greater than $70 \%$ in all 4 of the LMSC, averaging $84.0 \%$.

In terms of the square area of the nuclei (major axis $\times$ minor axis), that of the

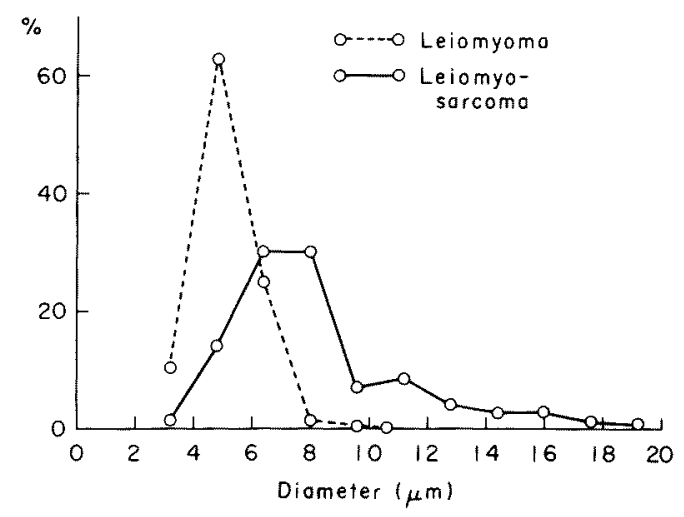

Fig. 1. Nuclear minor axis diameter Meansts.D. of the diameters in leiomyoma and leiomyosarcoma are $4.6 \pm 1.0$ and $7.5 \pm 2.7 \mu \mathrm{m}$, respectively. 
TABLE 1. Cytological findings of leiomyogenic tumors of the stomach

\begin{tabular}{|c|c|c|}
\hline & Leiomyoma & Leiomyosarcoma \\
\hline \multicolumn{3}{|l|}{ Nucleus } \\
\hline \multicolumn{3}{|l|}{ Major axis diameter $(\mu \mathrm{m})$} \\
\hline Mean value \pm S.D. & $18.0 \pm 5.6$ & $15.3 \pm 7.4$ \\
\hline Range of distribution & $5.6-38.4$ & $5.6-36.8$ \\
\hline \multicolumn{3}{|l|}{ Minor axis diameter $(\mu \mathrm{m})$} \\
\hline Mean value \pm s.D. & $4.6 \pm 1.0$ & $7.5 \pm 2.7$ \\
\hline Range of distribution & $2.4-10.4$ & $3.2-18.4$ \\
\hline $\begin{array}{l}\text { Percentage over } 5 \mu \mathrm{m} \\
\text { (mean value) }\end{array}$ & 25.796 & $84.0 \%$ \\
\hline \multicolumn{3}{|l|}{ Square area $\left(\mu \mathrm{m}^{2}\right)$} \\
\hline Mean value \pm s.D. & $82.8 \pm 26.7$ & $121.7 \pm 32.0$ \\
\hline \multicolumn{3}{|l|}{ Staining quality $(\%)$} \\
\hline Pale & 0 & 0 \\
\hline Normal & 72.9 & 18.4 \\
\hline Slightly dark & 26.7 & 60.8 \\
\hline Moderately dark & 0.4 & 20.8 \\
\hline Markedly dark & 0 & 0 \\
\hline \multicolumn{3}{|l|}{ Chromocenter pattern $(\%)$} \\
\hline $3 a$ or $3 a 4 c$ & 64.1 & 14.0 \\
\hline $3 a 4 c 5 c$ or $3 a 5 c$ & 19.4 & 19.8 \\
\hline $3 a 4 b$ or $3 a 5 b$ & 15.9 & 25.3 \\
\hline $4 a$ or $4 a 5 c$ & 0.6 & 31.5 \\
\hline $4 a 5 b$ or 5,6 & 0 & 9.4 \\
\hline \multicolumn{3}{|l|}{ Nuclear rim $(\%)$} \\
\hline R2 & 0.1 & 0 \\
\hline $\mathrm{R} 3$ & 49.7 & 10.8 \\
\hline $\mathrm{R} 34$ & 32.9 & 18.5 \\
\hline R4 & 16.9 & 38.1 \\
\hline $\mathrm{R} 4-5$ & 0.4 & 21.3 \\
\hline R5 & 0 & 11.3 \\
\hline \multicolumn{3}{|l|}{ Nuclear clear area $(\%)$} \\
\hline $\mathrm{N} 1+2$ & 6.0 & 10.3 \\
\hline $\mathrm{N} 2$ & 74.7 & 40.4 \\
\hline N $2+3$ & 19.3 & 32.5 \\
\hline N 3 & 0 & 8.8 \\
\hline $\mathrm{N} 3+4$ & 0 & 7.0 \\
\hline N 4 & 0 & 1.0 \\
\hline \multicolumn{3}{|l|}{ Nucleolus } \\
\hline \multicolumn{3}{|l|}{ Maximum diameter $(\mu \mathrm{m})$} \\
\hline Mean value & 0.9 & 1.9 \\
\hline Range of distribution & $0.6-1.6$ & $0.4-10.4$ \\
\hline \multicolumn{3}{|l|}{ Number } \\
\hline Mean value & 1.6 & 2.5 \\
\hline Range of distribution & $1-4$ & $1-30$ \\
\hline \multicolumn{3}{|l|}{ Type $(\%)$} \\
\hline Smooth & 18.7 & 24.8 \\
\hline Isolated & 4.0 & 14.5 \\
\hline Arborescent & 40.5 & 34.3 \\
\hline Small multiple & 0 & 1.5 \\
\hline Mixed & 22.1 & 19.7 \\
\hline Indistinet & 14.7 & 5.2 \\
\hline \multicolumn{3}{|l|}{ Nuclear shpe $(\%)$} \\
\hline Oval & 6.6 & 55.0 \\
\hline Long oval & 18.1 & 18.0 \\
\hline
\end{tabular}


TABLE 1. Continued

\begin{tabular}{lcc} 
& Leiomyoma & Leiomyosarcoma \\
\hline Vienna sausage form & 74.3 & 17.8 \\
Others & 1.0 & 9.2 \\
Nuclear outline (\%) & & \\
Smooth & 76.7 & 67.3 \\
Irregular & & \\
$\quad$ Notched & 5.9 & 15.0 \\
Sharply-clefted & 11.8 & 9.5 \\
Nipple-like tubercular & 5.6 & 7.5 \\
Concave & 0 & 0.7 \\
Cytoplasm & & \\
Cell shape (\%) & & 13.6 \\
Long oval & 0 & 5.7 \\
Fiber & 61.3 & 51.1 \\
Spindle & 22.3 & 16.1 \\
Tadpole & 7.0 & 13.5 \\
Others & 9.4 & \\
Staining quality of cytoplasm (\%) & & 65.3 \\
Basophilic palely & 100.0 & 6.3 \\
Polychromatic palely & 0 & 25.0 \\
& 0 & 3.4 \\
\hline
\end{tabular}

LMSC was $121.7 \pm 32.0 \mu^{2} \mathrm{~m}$, which was significantly greater than that of LMC $\left(82.8 \pm 26.7 \mu \mathrm{m}^{2}\right)$, and the former showed a much wider distribution range.

\section{Staining quality of the nuclei}

Staining of the nuclei was classified into five groups: pale, normal, slightly dark, moderately dark and markedly dark. The majority of LMC showed normal staining $(72.9 \%$ ), whereas only a small percentage $(27.1 \%$ ) showed slightly or moderately dark staining. LMSC, on the other hand, showed normal staining in only $18.4 \%$ and greater than $80 \%$ showed dark staining $(60.8 \%$ slightly dark and $20.8 \%$ moderately dark). The darkness and the unevenness of the nuclear staining were marked.

\section{Nuclear structure}

An investigation of nuclear structure was undertaken in the components of the chromocenter, nuclear rim, nuclear clear area and the nucleolus.

Chromocenter. The chromocenter (C) was classified into 7 groups according to size, shape and staining quality: powdered (C1), palely-stained granular (C2), normally-stained granular (C3), deeply-stained granular (C4), small clumpy (C5), medium clumpy (C6), and large clumpy (C7). A further classification was made in terms of the number of chromocenters: a large number $(a)$, a moderate number $(b)$, and a small number $(c)$ (Kobiyama et al. 1973). In this manner, the chromocenter pattern of each nucleus could be expressed as, for example, $3 a 4 c$ (that is a nucleus with a large number of $\mathrm{C} 3$ chromocenters and a small number of $\mathrm{C} 4$ chromocenters) (Table 1). In cases of LMC, the primary cell type was $3 a 4 c$-in other words, 
nuclei with C3 normally-stained granular chromocenters were found in $64.1 \%$ of the cells. In contrast, in LMSC, nuclei with $\mathrm{C} 4$ deeply-stained granular chromocenters were the most abundant (31.5\%) and C5 and C6 nuclei with clumpy chromocenters accounted for $9.4 \%$. That is, in comparison with LMC, LMSC had a high incidence of deeply-stained or clumpy chromocenters (Figs. 2 and 3).

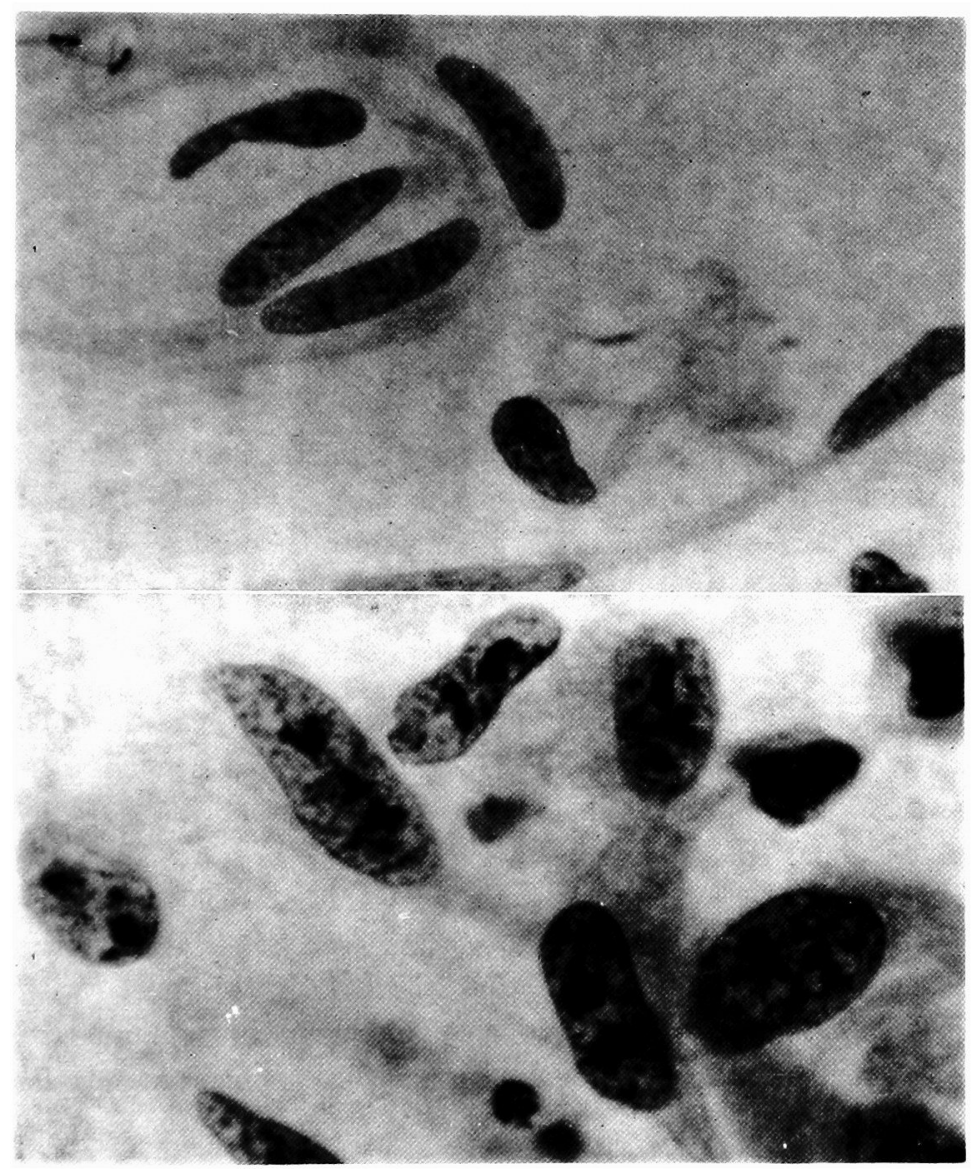

Fig. 2 (upper). Leiomyoma cells (Papanicolaou staining $\times 1000$ ). Normally-stained granular chromocenters (C3) are evenly distributed within the nuclei. Nuclear rims are smooth with no thickening.

Fig. 3 (lower). Leiomyosarcoma cells $($ Pap $\times 1000)$. A large amount of deeply-stained granular chromocenters (C4) and small clumpy chromocenters (C5) are found in the nuclei. Some of the nuclear rims are seen to be irregularly thickened.

Nuclear rim. Depending upon the difference in staining and thickness of the nuclear outline, the nuclear rim was also classified into 7 categories, R1 through R7. This classification roughly correlated with that of the chromocenter. In LMC, R3 (which corresponds to the thickness and degree of staining of C3) was the most frequent nuclear rim pattern $(49.7 \%$ ), whereas R4 and R5 were found at a low 
incidence (17.3\%). In contrast, in LMSC, R4 (which corresponds to the thickness and degree of staining of $\mathrm{C} 4$ ) was the most frequent $(38.1 \%)$, with R45 or R5, moderate rim thickening, being found in about $33 \%$.

Nuclear clear area. The clear areas located between the chromocenters and chromatin bands were termed nuclear clear areas (N) and a 5 group classification made: minute (N1), small (N2), medium (N3), large and delicately demarcated (N4), and large and heavily demarcated (N5). Mixed types were expressed as $\mathrm{N} 2+3$, etc. In LMC, $\mathrm{N} 2$ totalled $74.7 \%$ and atypical patterns were infrequent, whereas in LMSC, the incidence of $\mathrm{N} 2+3, \mathrm{~N} 3, \mathrm{~N} 3+4$ and $\mathrm{N} 4$ nuclei with enlarged clear areas had greatly increased.

Nucleolus. The average number of nucleoli per cell in LMC was 1.6 , more than $90 \%$ of the LMC cell having 1 or 2 . In LMSC cases, the average number was 2.5 with a wide distribution, and $15 \%$ of them were with more than 5 nucleoli.

In LMC, the maximum nucleolar diameter (in nuclei with more than one nucleolus, the maximum diameter of the largest nucleolus) averaged $0.9 \mu \mathrm{m}$, whereas it was $1.9 \mu \mathrm{m}$ in LMSC.

A classification of nucleolar type was made into the following 5 types: smooth, isolated, arborescent, small-multiple, and mixed. Both LMC and LMSC had predominantly arborescent type nucleoli, but the incidence of the isolated type was relatively high in LMSC.

\section{Nuclear shape}

The incidence of various nuclear shapes in LMC and LMSC is shown in Table 1. Round, oval, long oval and Vienna sausage forms were defined in terms of the major axis diameter/minor axis diameter ratio $(R)$ : round, $R<1.2$; oval, $1.2<R$ $<2.0$; long oval, $2.0<R<3.0$; and Vienna sausage form, $R>3.0$. It was found that in LMC the great majority cells $(74.3 \%)$ had Vienna sausage type nuclei, whereas in LMSC greater than half $(55 \%)$ had oval nuclei and Vienna sausage nuclei accounted for only $17.8 \%$. In other words, long, thin nuclei with blunt end were typical of LMC (Fig. 4), whereas due to an increase in the minor axis diameter in LMSC, the incidence of round or oval nuclei was increased and pleomorphism was marked (Fig. 5).

\section{Nuclear outline}

The nuclear outline was grouped as smooth or irregular, and the irregular type was further classified into notched, sharply clefted, nipple-like tubercular and concave. It was seen that cells with a smooth nuclear outline were most numerous in both LMC and LMSC $(>50 \%)$, but the incidence of irregularly outlined nuclei was somewhat higher in LMSC than in LMC.

\section{Cell shape}

The majority of both LMC and LMSC showed long, thin shapes (spindle-shaped, fiber-shaped or tadpole-shaped), but LMC showed predominantly fiber-shaped 


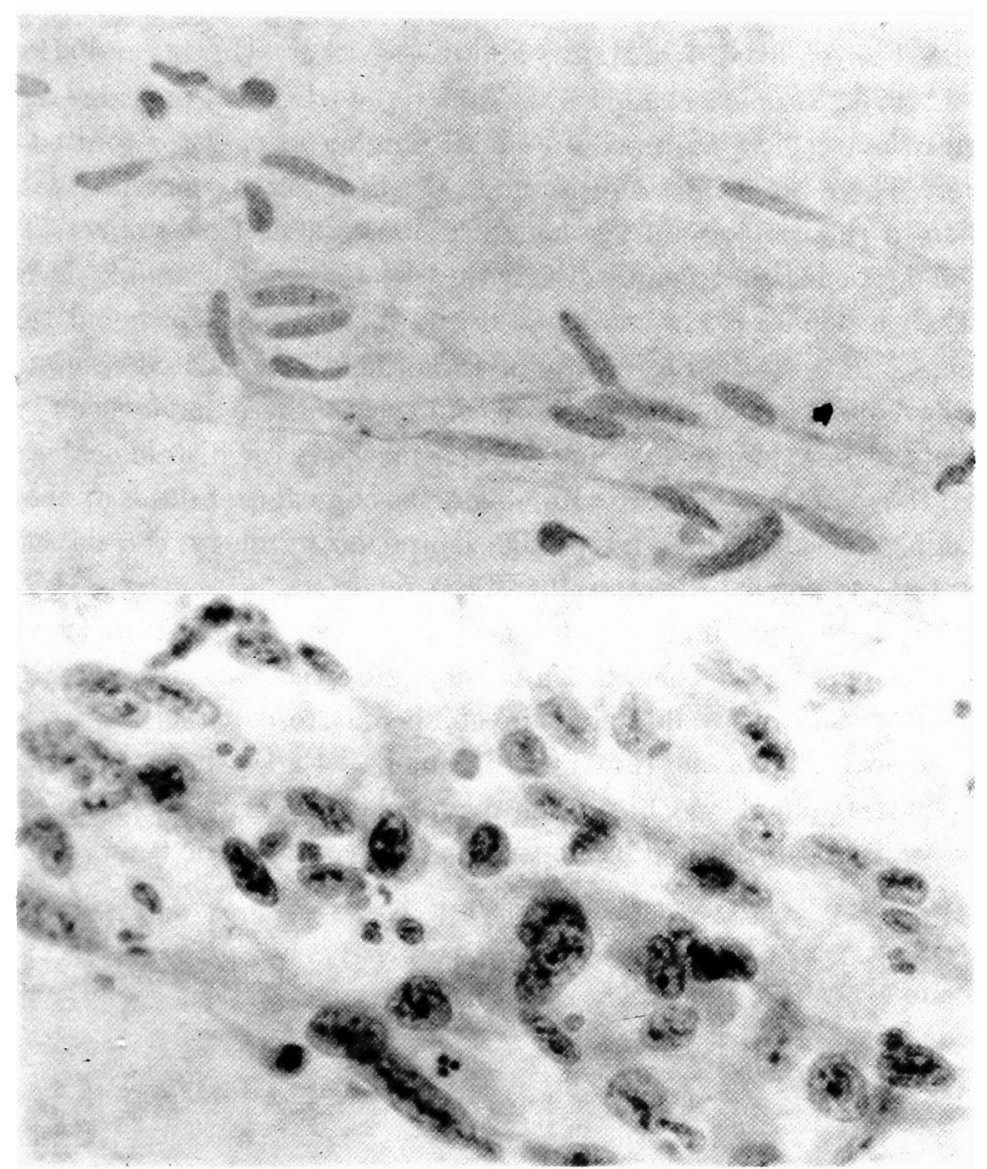

Fig. 4 (upper). Leiomyoma cells (Papanicolaou staining $\times 200$ ). The predominant nuclear shape is the Vienna sausage form with blunt ends; slight anisokaryosis and pleomorphism are seen.

Fig. 5 (lower). Leiomyosarcoma cells (Papanicolaou staining $\times 200$ ). The predominant nuclear shapes are round and oval; marked anisokaryosis and pleomorphism are seen.

$(61.3 \%)$, whereas LMSC showed a high incidence $(51.1 \%)$ of the relatively fat spindle-shape. Furthermore, LMSC was frequently long oval, etc., showing a high incidence of pleomorphism.

\section{Staining quality of the cytoplasm}

Fully $100 \%$ of the LMC showed basophilic staining, whereas, although a majority of the LMSC were basophilic, roughly $30 \%$ were polychromatic, including a high incidence of anioschromatism.

\section{Discussion}

There have been few reports on cytological findings in leiomyogenic tumors of 
the stomach and even in the reports describing the cytology of malignant leiomyosarcoma (Cabre-Fiol et al. 1975; Hajdu and Koss 1969; Hajdu and Hajdu 1976; Shida et al. 1970; Yamada et al. 1973; Yamada et al. 1974) most are limited to a description of cytological findings of a small number of cases or limited to general statements. There are in fact no reports in the literatures concerned with detailed examination of the cytology of the benign lesion, gastric leiomyoma. The lack of research on non-epithelial tumors, including leiomyogenic tumors, is due to the fact that their incidence is low compared to the incidence of epithelial tumors such as carcinoma and a method for sampling tumorous tissue located submucosally has not been developed. Nonetheless, in recent years there have been reports of developments of new biopsy instruments and methods for histological sampling of sumbucosal tissue of the stomach. Moreover, the clinical usefulness of thorny needle biopsy (Ishioka et al. 1979) and needle aspiration cytology (Soeda et al. 1976) for differential cytological diagnosis has been reported.

Using the thorny needle biopsy method, developed at the Third Department of Internal Medicine of the Tohoku University Hosptial, a submucosal tumor is biospsied with a thorny needle under direct fibergastroscopic vision and diagnosis of the histological type of the tumor can be made. Fig. 6 shows the entire thorny needle biopsy instrument and Fig. 7 shows an enlargement of the tip. Since April, 1974, we have used this method in biopsy of submucosal gastric tumors in 185 cases,
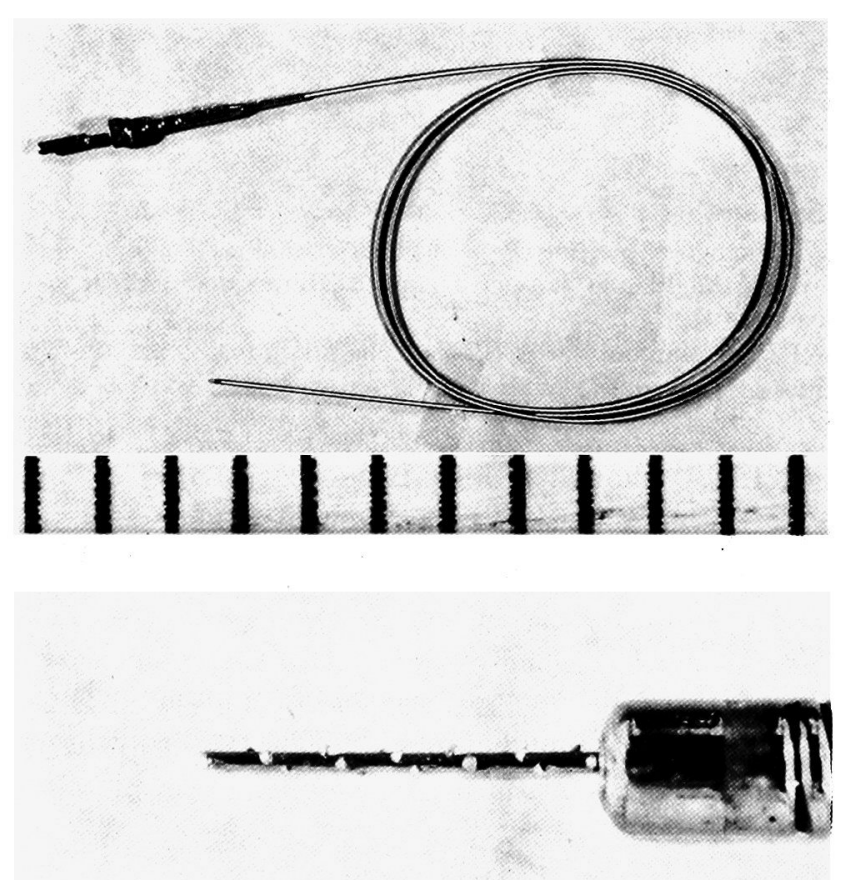

Fig. 6 (upper). The entire thorny needle biopsy instrument.

Fig. 7 (lower). An enlargement of the thorny needle. 
12 of which were diagnosed as gastric leiomyogenic. The rates of tumor cell sampling using this method are summarized in Table 2. All 4 of the leiomoyosarcoma and 6 of the 8 lieomyomas were biopsied using these methods. Moreover, it has proven possible to obtain tumor cells in 4 of the 6 cases in which no ulceration of the mucosa of the tumor was apparent. Since only the cells within the mucosa can be sampled using the usual biopsy instrument, but almost none of the submucosal layer, the advantages of the thorny needle biopsy method are apparent.

TABLE 2. Rate of tumor cell sampling using the thorny needle biopsy method

\begin{tabular}{lcccccc}
\hline Histological type & Number of cases & \multicolumn{2}{c}{ Ulceration $(-)$} & & \multicolumn{2}{c}{ Ulceration (+) } \\
\cline { 3 - 4 } \cline { 5 - 6 } & $\begin{array}{c}\text { Number of } \\
\text { cases }\end{array}$ & $\begin{array}{c}\text { Rate of } \\
\text { cytological } \\
\text { sampling }\end{array}$ & $\begin{array}{c}\text { Number of } \\
\text { cases }\end{array}$ & $\begin{array}{c}\text { Rate of } \\
\text { cytological } \\
\text { sampling }\end{array}$ \\
\hline Leiomyosarcoma & 4 & 1 & $1 / 1$ & 3 & $3 / 3$ \\
$\begin{array}{l}\text { Leiomyoma } \\
\text { Total }\end{array}$ & 8 & 5 & $3 / 5$ & 3 & $3 / 3$ \\
\hline
\end{tabular}

Classification is made according to whether or not ulceration of the surface of the tumor was seen.

Owing to the development of this new biopsy method, sampling of submucosal tumor cells has become possible and the need for statistical research on the cytology of various tumors based upon cytological diagnosis has arisen. From this point of view, we have undertaken an investigation of 11 cases of leiomyogenic tumors of the stomach (7 cases of leiomyoma and 4 cases of leiomyosarcoma), which are known to have a relatively high incidence among the submucosal gastric tumors and for which determination of their malignancy is a clinically important problem.

As summarized in Table 1, we have found that the cytological differential diagnosis of leiomyoma and leiomyosarcoma is indeed possible. Similar to other submucosal lesions of the stomach, it has long been extremely difficult to make a preoperative diagnosis of leiomyogenic tumors of the stomach, so that nearly all definitive diagnosis has been possible only at autopsy or from histopathological examination of surgically excized tissue. There are, however, numerous problems remained on the histological criteria used in determining the malignancy of these lesions and, as Nagata et al. (1970) and Shida et al. (1975) have indicated, there is some confusion concerning what histological criteria should be used. Stout and Hill (1958) maintain that the number of mitoses is important in determining the degree of malignancy of leiomyogenic tumors. Sano et al. (1970) supported this view with regard to determining the malignancy of leiomyosarcoma, and found no mitosis in benign leiomyoma. On the other hand, Giberson et al. (1954) reported that in 15 of 40 cases of leiomyosarcoma of the stomach there was almost no sign of mitosis. Kubo et al. (1965) pointed out that the belief that the prognosis for patients with leiomyosarcoma is generally better than that of other malignant tumors is due to the frequent histological misdiagnosis of benign leiomyomas as 
malignant leiomyosarcomas. France and Brines (1950) and Sano et al. (1970) have also indicated that, due to the frequent nuclear palisading of gastric leiomyogenic tumors, they are frequently misdiagnosed as neurilemmoma. Because of the above-mentioned confusion concerning the histological diagnostic criteria for leiomyogenic tumors of the stomach, it is thought that even using the newly developed biopsy methods, which now allow for sampling sufficient submucosal tissue, some problems in making the final determination still remain.

In the current study, clear differences in the cytology of leiomyomas and leiomyosarcomas of the stomach have been found. This fact indicates that, using the thorny needle biopsy method and aspiration cytology, differential diagnosis should be possible provided that a sufficient number of tumor cells are sampled. Henceforth, we intend to collect a more cases and, by further clarifying the cytological diagnostic criteria, more precise preoperative diagnosis with be made possible on leiomyogenic tumors of the stomach.

\section{References}

1) Cabre-Ficol, V., Vilandeli, F., Sala-Cladera, E. \& Mota, A.P. (1975) Preoperative cytological diagnosis of gastric leiomyosarcoma. Gastroenterology, 68, 563-566.

2) France, C.J. \& Brines, O.A. (1950) Mesenchymal tumors of the stomach. Arch. Surg., 61, 1019-1035.

3) Giberson, R.G., Dockerty, M.B. \& Gray, H.K. (1954) Leiomyosarcoma of the stomach. Surg. Gynec. Obstet., 98, 186-196.

4) Hajdu, S.I. \& Hajdu, E.O. (1976) Leiomyosarcoma. In: Cytopathology of Sarcomas and Other Nonephithelial Malignant Tumors, edited by S.I. Hajdu \& E.O. Hajdu, W.B. Saunders Co., Philadelphia-London-Toront, pp. 199-208.

5) Hajdu, S.I. \& Koss, L.G. (1969) Cytologic diagnosis of metastatic myosarcomas. Acta cytol., 13, 545-551.

6) Ishioka, K., Sato, A., Umetsu, Y. Toyohara, T. \& Kobiyama, M. (1979) Limitations of cytological diagnosis for early gastric cancer. Stomach and Intestine, 14, 173-182. (Japanese).

7) Kobiyama, M., Yamagata, S., Ishioka, K., Ueno, K. Goto, M. \& Sato, A. (1973) Cytological observation of nucleus of atypical epithelium of the stomach: with special reference to differential diagnosis of early cancer. Jap. J. Gastroent., 70, 1206-1211. (Japanese)

8) Kubo, T. Nakao, K. \& Harasawa, T. (1965) Bizarre leiomyoblastoma of the stomach. Jap. J. Cancer Clin., 11, 643-646. (Japanese)

9) Nagata, K., Hanashi, K., Ueno, K., Niino, K., Sugaya, H., Miyamoto, T. \& Furuuchi, M. (1970) A case of leiomyoma in the cardiac region. Stomach and Intestine, 5, 15211525. (Japanese)

10) Sano, R., Hirota, E., Shimoda, T., Yamamoto, H. \& Watanabe, H. (1970) Pathology of gastric sarcoma. Stomach and Intestine, 5, 311-322. (Japanese)

11) Shida, S., Sawada, Y., Yasui, A., Takamura, T., Momo, S., Matsuzawa, Y., Ikeguchi, S., Hayashi, H. \& Shimoda, M. (1970) Cytological diagnosis of gastric sarcoma. Stomach and Intestine, 5, 301-310. (Japanese)

12) Shida, S., Nagashima, K., Arakawa, M., Matsuzawa, Y., Ikeguchi, S., Yøkota, K., Muto, K., Hidaka, T. \& Takada, E. (1975) Non-epithelial tumor of the gastrointestinal tract - Its clinical study -. Stomach and Intestine, 10, 861-876. (Japanese)

13) Soeda, S., Kondo, M., Akashi, M., Ito, T., Tsuchiya, R. \& Futatsugi, K. (1976) Aspiration biopsy of the stomach: Efficient procedure for evaluating submucosal tumor. Stomach and Intestine, 11, 425-430. (Japanese) 
14) Stout, A.P. \& Hill, W.T. (1958) Leiomyosarcoma of the superficial soft tissues. Cancer, 11, 844-854.

15) Yamada, T., Kakihana, M., Kosaki, I., Tabayashi, A., Murohisa, B. \& Doi, K. (1973) Exfoliative cytology on malignant neoplasms of the non-epithelial origin: Cytologic morphology of myogenic sarcomas. J. Jap. Soc. clin. Cytol., 12, 77-83. (Japanese)

16) Yamada, T., Kakihana, M. \& Kosaki, I. (1974) Exfoliative cytology of the nonephithelial tumors with special references to their cyomorphologic bases. J. Jap. Soc. clin. Cytol., 13, 139-155. (Japanese) 